\title{
Implementing Rotation Forest for Wind Turbine Blade Fault Diagnosis
}

\author{
A. Joshuva, G. Deenadayalan, S. Sivakumar, \\ R. Sathishkumar, R. Vishnuvardhan
}

\begin{abstract}
Wind energy is one of the important renewable energy resources because of its reliability due to the development of the technology and relative less cost. The wind energy are converted into electrical energy using rotating blades which are connected to the generator. Due to environmental conditions and large structure, the blades are subjected to various faults and cause the lack of productivity. The downtime can be reduced when they are diagnosed periodically using structural health monitoring. These are considered as a pattern recognition problem which consist of three phases, namely feature extraction, feature selection and feature classification. In this research, statistical features are extracted from vibration signals, feature selection are carried out using $\mathrm{J48}$ algorithm and the feature classification is done with a rotation forest algorithm.
\end{abstract}

Keywords--- Fault Diagnosis, Structural Health Monitoring, Statistical Features, J48 Algorithm, Rotation Forest (RF) Algorithm.

\section{INTRODUCTION}

Wind energy turns into the most requested resource in present era because of universal environmental contagion [1]. Wind energy is one of the proficient renewable sources and another choice for normally used sources. Wind turbine are used to convert wind energy into electrical energy. To make wind energy more focused from different sources of energy, like performance, accessibility, reliability, effectiveness, the life of turbines must be improved [2]. The wind makes the rotor cutting edge turn, thus making the pole inside the wind turbine turn. The flimsy, long and malleable sharp edge is the part suffers the most extreme complex forces. It is easy to initiate vibration [3]. The rehashed and severe vibration will prompt damage to the cutting edge and last to failure. The cutting edge is one of the vital parts with rehashed failure. Due to its massive construction and functioning condition, the vibration of the cutting edge is challenging to compute on-line. In this study, fault diagnosis on wind turbine blade is carried out using vibration signals through machine learning approach.

A data-driven approach for monitoring blade pitch faults in wind turbines was carried out by Andrew Kusiak and

\footnotetext{
Manuscript received September 16, 2019.

A. Joshuva*, Centre for Automation and Robotics (ANRO), Department of Mechanical Engineering, Hindustan Institute of Technology and Science, Old Mahabalipuram Road, Padur, Kelambakam, Chennai, Tamil Nadu. (e-mail: joshuva1991@gmail.com)

G. Deenadayalan, Centre for Automation and Robotics (ANRO), Department of Mechanical Engineering, Hindustan Institute of Technology and Science, Old Mahabalipuram Road, Padur, Kelambakam, Chennai, Tamil Nadu.

S. Sivakumar, Department of Mechanical Engineering, Hindustan Institute of Technology and Science, Old Mahabalipuram Road, Padur, Kelambakam, Chennai, Tamil Nadu.

R. Sathishkumar, Department of Automobile Engineering, Hindustan Institute of Technology and Science, Old Mahabalipuram Road, Padur, Kelambakam, Chennai, Tamil Nadu.

R. Vishnuvardhan, Department of Mechatronics Engineering, Sri Krishna College of Engineering and Technology, Coimbatore, Tamil Nadu.
}

Anoop Verma [4]. They used bagging, artificial neural network (ANN), pruning rule based classification tree (PART), K-nearest neighbour (K-NN) and genetic programming (GP) for classification of the fault.

The accuracy was found to be GP-74.7\%, Bagging$72.5 \%, \quad$ PART-75.5\%, ANN-76.2\%, K-NN-73.5\% Classification and detection of wind turbine pitch faults through SCADA data Analysis using Repeated Incremental Pruning to Produce Error Reduction (RIPPER) classifier was studied by Godwin and Mathew [5]. They obtained the classification accuracy of $87.05 \%$. Frost et. al [6] carried out a study on integrating structural health management with contingency control for wind turbines using nonlinear highfidelity simulation. They obtained the classification accuracy of $90 \%$.

A study on wavelet transform based stress and time history editing of horizontal axis wind turbine blades was carried out by Pratumnopharat et. al [7]. They used time correlated fatigue damage (TCFD), mexican hat wavelet (Mexh), meyer wavelet (Meyr), daubechies 30th order (DB30), morlet wavelet (Morl), discrete meyer wavelet (Dmey) for the classification of crack on blade. The accuracy they found to be TCFD-89.82\%, Morl-80.34\%, Meyr-79.76\%, Dmey-80.30\%, Mexh-79.23\%, DB30$80.81 \%$.

Design of shape-adaptive wind turbine blades using differential stiffness bend-twist coupling (DSBT) was studied by Herath et. al [8]. The concept is proposed to control the deformation behaviour of the blades using FEM analysis. Wang Yongzhi et. al [9] have carried out a study on composite wind turbine blade aerodynamic and structural integrated design optimization based on radial basis function (RBF) meta-model, blade element momentum (BEM) theory, finite element method (FEM) and multi-island genetic algorithm (MIGA). This paper aims to reduce the mass of blade under some constraints, including the power and deflection at the rated wind speed, and the strength and deflection under ultimate case.

Many works where carried out using simulation work and very few in experimental analysis [10]. Machine learning approach was very much less considered for wind turbine blade fault diagnosis and only very few faults were considered. This study makes an attempt to do diagnosis of various blade defects using machine learning approach (statistical features and rotation forest classifier). Vibration signals are taken from the blades using uni-axial accelerometer. Figure 1 shows the methodology of the work done. 


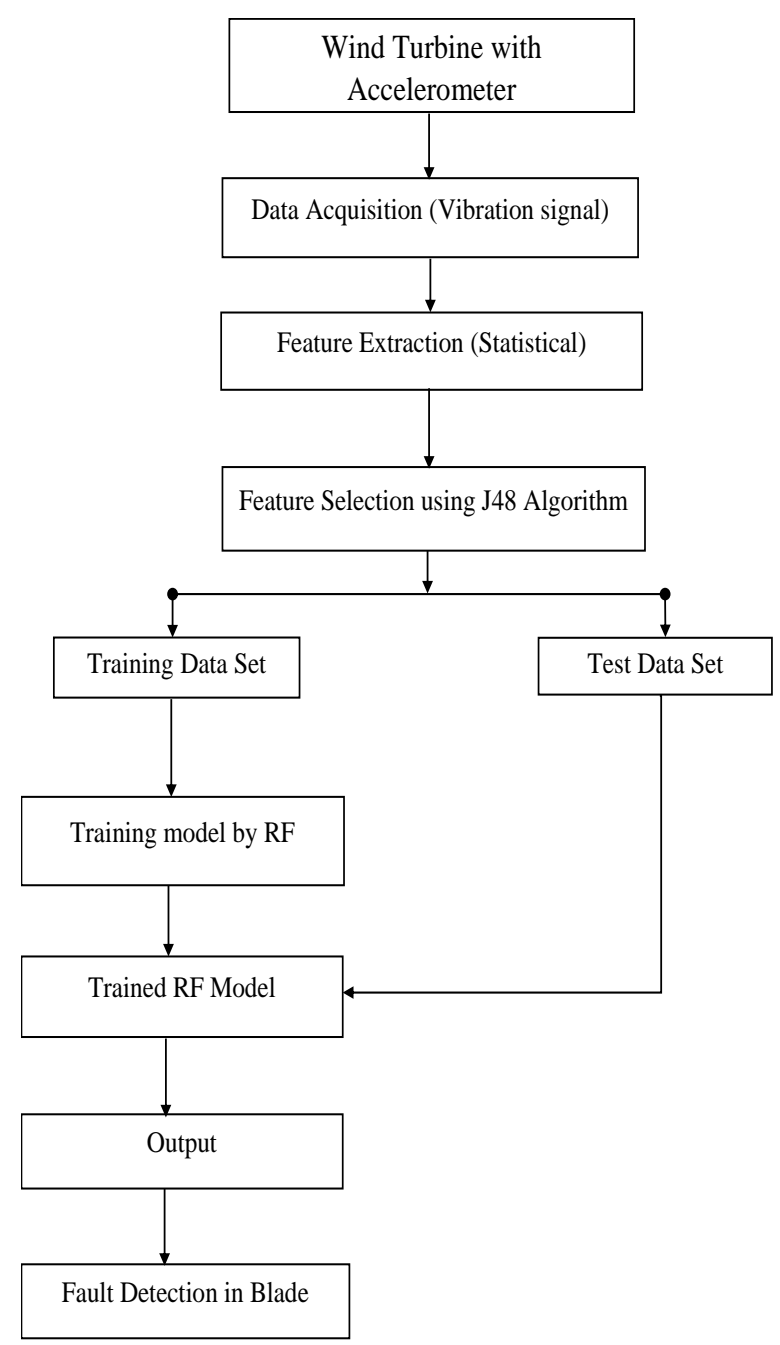

Figure 1: Methodology

\section{EXPERIMENTAL STUDIES}

The main aim of this study is to classify whether the blades are in good condition or in a defective state. "If it is defective, then the objective is to identify the type of fault.

The experimental setup and experimental procedure are described in the following subsections.

\section{Experimental Setup}

The experiment was carried out on a $50 \mathrm{~W}, 12 \mathrm{~V}$ variable speed wind turbine (MX-POWER, model: FP-50W-12V) [11]. The technical parameters of a wind turbine are given in Table 1.

The wind turbine was mounted on a fixed steel stand in front of the open circuit wind tunnel outlet. The wind tunnel speed ranges from $5 \mathrm{~m} / \mathrm{s}$ to $15 \mathrm{~m} / \mathrm{s}$ and acts as a wind source to start the wind turbine. The wind speed was varied continuously in order to simulate the environmental wind condition.

The experimental setup is shown in Figure 2. Piezoelectric type accelerometer was used as a transducer for acquiring vibration signals. It has high sensitivity for detecting faults.

Hence, accelerometers are widely used in condition monitoring. In this case, an uniaxial accelerometer of $500 \mathrm{~g}$ range, $100 \mathrm{mV} / \mathrm{g}$ sensitivity, and resonant frequency around $40 \mathrm{~Hz}$ was used."
Table 1: Technical Parameters of Wind Turbine

\begin{tabular}{|l|l|}
\hline Model & FP-50W-12V \\
\hline Rated Power & $50 \mathrm{~W}$ \\
\hline Rated Voltage & $12 \mathrm{~V}$ \\
\hline Maximum Current & $4 \mathrm{~A}$ \\
\hline $\begin{array}{l}\text { Rated Rotating } \\
\text { Rate }\end{array}$ & $850 \mathrm{rpm}$ \\
\hline $\begin{array}{l}\text { Start- } \\
\text { up Wind Speed }\end{array}$ & $2.5 \mathrm{~m} / \mathrm{s}$ \\
\hline Cut-in Wind Speed & $3.5 \mathrm{~m} / \mathrm{s}$ \\
\hline $\begin{array}{l}\text { Cut- } \\
\text { out Wind Speed }\end{array}$ & $15 \mathrm{~m} / \mathrm{s}$ \\
\hline $\begin{array}{l}\text { Security Wind Spee } \\
\text { d }\end{array}$ & $40 \mathrm{~m} / \mathrm{s}$ \\
\hline Rated Wind Speed & $12.5 \mathrm{~m} / \mathrm{s}$ \\
\hline Engine & $\begin{array}{l}\text { Three- } \\
\text { phase permanent magnet generat } \\
\text { or }\end{array}$ \\
\hline Rotor Diameter & $1050 \mathrm{~mm}$ \\
\hline Blade Material & Carbon fiber reinforced plastics \\
\hline
\end{tabular}

The piezoelectric accelerometer (DYTRAN 3055B1) was mounted on the nacelle near to the wind turbine hub to record the vibration signals using an adhesive mounting technique. "It was connected to the DAQ system through a cable. The data acquisition system (DAQ) used was NI USB 4432 model.

The DAQ card has five analogue input channels with a sampling rate of 102.4-kilo samples per second with 24-bit resolution. The accelerometer is coupled to a signal conditioning unit which consists of an inbuilt charge amplifier and an analogue-to-digital converter (ADC). From the ADC, the vibration signal was taken.

These vibration signals were used to extract features through feature extraction technique. One end of the cable is plugged to the accelerometer and the other end to the AIO port of DAQ system. NI - LabVIEW was used to interface the transducer signal and the system (PC)."

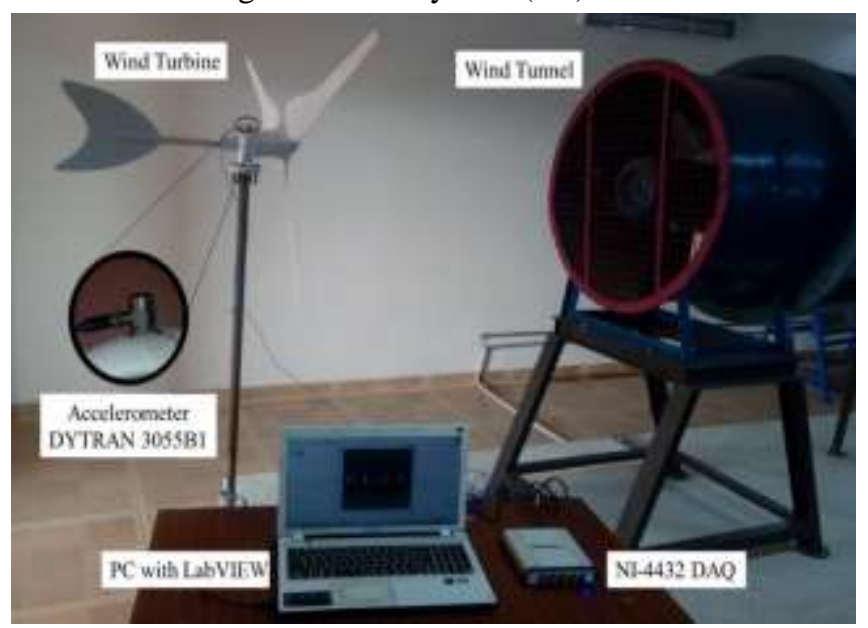

Figure 2: Wind Turbine Setup

\section{Experimental Procedure}

In the present study, three-blade variable horizontal axis wind turbine (HAWT) was used. "Initially, the wind turbine was considered to be in good condition (free from defects,

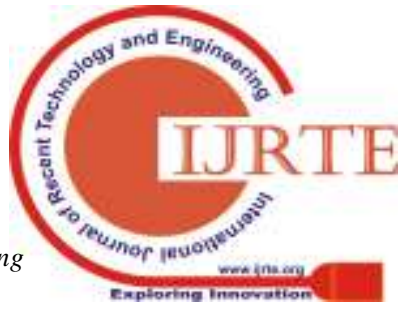


new setup) and the signals were recorded using an accelerometer. These signals were recorded with the following specifications.

1. Sample length: The sample length was chosen long enough to ensure data consistency; and also the following points were considered. ARMA measures are more meaningful when the number of samples is sufficiently large. On the other hand, as the number of samples increases the computation time increases. To strike a balance, a sample length of 10000 was chosen.
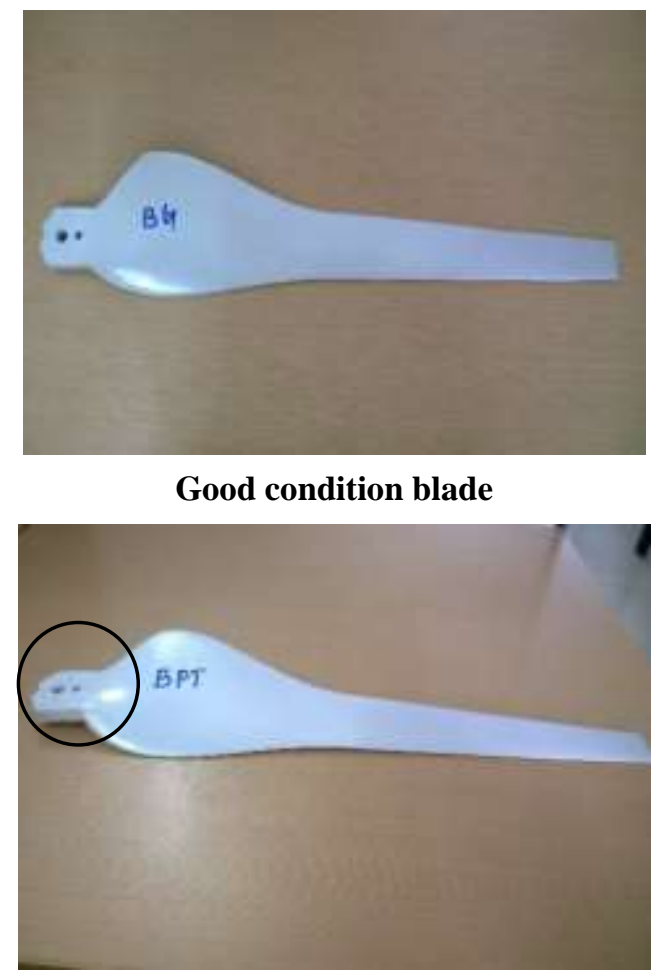

Blade with pitch angle twist

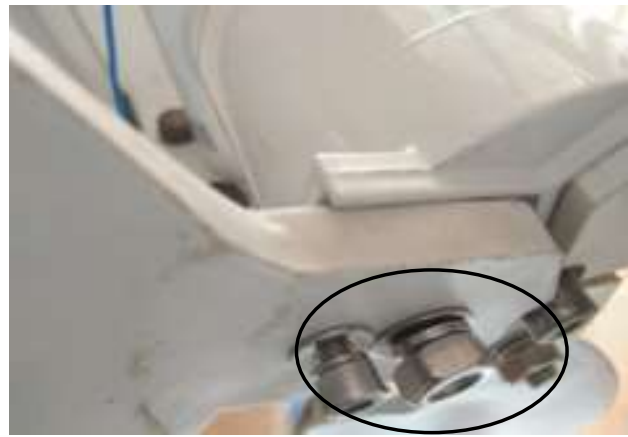

Hub-blade loose connection
2. Sampling Frequency: The sampling frequency should be at least twice the highest frequency contained in the signal as per Nyquist sampling theorem. By using this theorem sampling frequency was calculated as $12 \mathrm{kHz}(12000 \mathrm{~Hz})$.

3. Number of samples: Minimum of 100 (hundred) samples were taken for each condition of the wind turbine blade and the vibration signals were stored in data files.

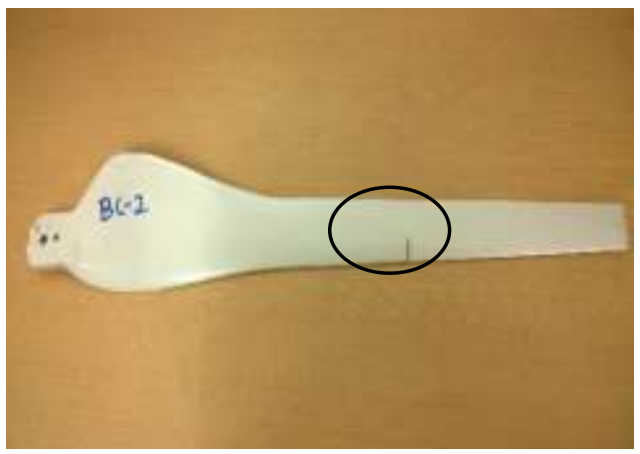

Blade with crack

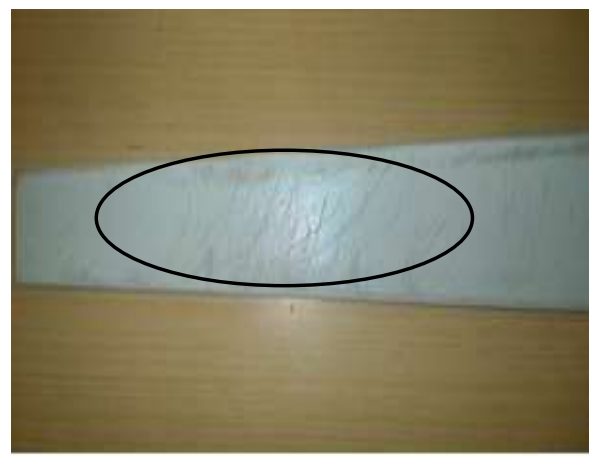

Blade with erosion

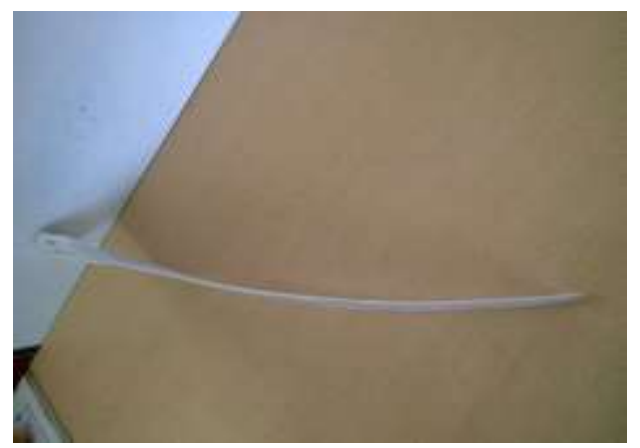

Blade with bend (Top View)

Figure 3: Various Blade Fault Conditions

The following faults were simulated one at a time while all other components remain in good condition and the corresponding vibration signals were acquired.

Figure 3 shows the different blade fault conditions which are simulated on the blade.

a) Blade bend (BB): This fault occurs due to the high-speed wind and complex forces caused by the

wind. The blade was made to flap wise bend with $10^{\circ}$ angle.

b) Blade crack (BC-2): This occurs due to foreign object damage on the blade while it is in operating condition. On the blade, $15 \mathrm{~mm}$ crack was made. 

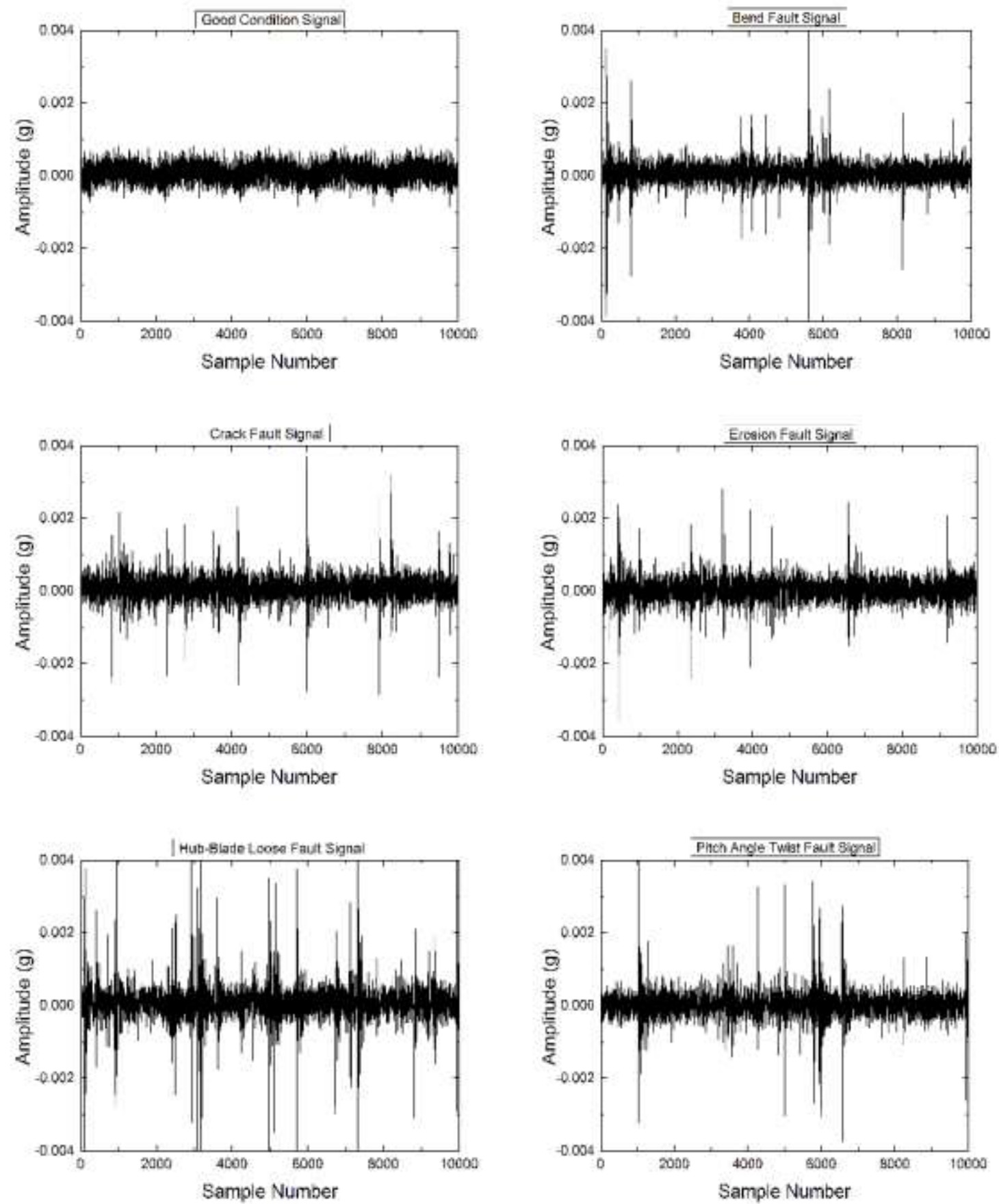

Figure 4: Vibration Signal Plot

c) Blade erosion (BE): This fault is due to the erosion of the top layer of the blade by the highspeed wind. The smooth surface of the blade was eroded using emery sheet $(320 \mathrm{Cw})$ to provide an erosion effect on the blade.

d) Hub-blade loose contact: This fault generally occurs on a wind turbine blade due to an excessive runtime or usage time. The bolt connecting the hub and blade was made loose to obtain this fault.

e) Blade pitch angle twist (PAT): This fault occurs due to the stress on the blade caused by high-speed wind. This makes the pitch get twisted, creating a heavy vibration to the framework. To attain this fault, blade pitch was twisted about $12^{\circ}$ angle with respect to the normal blade condition.

In Figure 4, the vibration signals (sample number vs. amplitude) are shown which were taken for different conditions of the wind turbine blade (good condition blade, blade bend, blade erosion, hub-blade loose connection, blade crack and pitch angle twist)."

\section{FEATURE EXTRACTION USING STATISTICAL ANALYSIS}

The vibration signals were attained for good and other defective conditions of the cutting edge. "If the time domain sampled signals are given directly as inputs to a classifier, then the number of samples should be constant. The number of samples attained which are the purposes of revolution of the cutting edge speed. Thus, it differs with the

\section{Published By:}

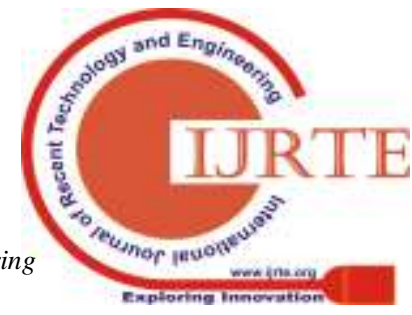


various speeds. Additionally, the capacity of digitized data points on a signal is too extensive. Usually, the work of classifiers may not handle it proficiently. So, a limited features need be extracted formerly the classification process. Descriptive statistical parameters [12] such as sum, mean, median, mode, minimum, maximum, range, skewness, kurtosis, standard error, standard deviation and sample variance were work out to assist as features in the feature extraction procedure.

- Sum: It is the sum of all feature values for each sample.

- Mean: The arithmetic average of a set of values or distribution.

- Median: Middle value sorting out the greater and lesser splits of a data set.

- Mode: Most frequent value available in the data set.

- Minimum value: It refers to the least signal point value in a given signal.

- Maximum value: It refers to the extreme signal point value in a given signal.

- $\quad$ Range: Difference in extreme and least signal point values for a given signal.

- Skewness: Skewness illustrates the degree of irregularity of a distribution around its mean. The following formula was used for calculation of skewness.

$$
\text { Skewness }=\frac{n}{(n-1)(n-2)} \sum\left(\frac{x_{i}-\bar{x}}{s_{d}}\right)^{3}
$$

- Kurtosis: Kurtosis point toward the flatness or the spikiness of the signal. Its value is very low for normal condition of the blade and high for the faulty condition of the blade due to the spiky nature of the signal and ' $\mathrm{s}$ ' is the sample standard deviation

$$
\text { Kurtosis }=\left\{\frac{n(n+1)}{(n-1)(n-2)(n-3)} \sum\left(\frac{x_{i}-\bar{x}}{s_{d}}\right)^{4}\right\}-\frac{3(n-1)^{2}}{(n-2)(n-3)}
$$

- Standard error: Standard error is a measure of the amount of error in the prediction of $y$ for an individual $\mathrm{x}$ in the regression, where $\mathrm{x}$ and $\mathrm{y}$ are the sample means and ' $n$ ' is the sample size.

Standard Error $(y)=\sqrt{\frac{1}{n-2}\left[\sum(y-\bar{y})^{2}-\frac{\Sigma[(x-\bar{x})(y-\bar{y})]^{2}}{\sum(x-\bar{x})^{2}}\right]}$

- Standard deviation: This is a measure of the actual energy or power content of the vibration signal. The following formula was used for calculation of standard deviation.

- Sample variance: It is the variance of the signal points and the following formula was used for calculation of sample variance.

$$
\text { Sample Variance }=\frac{n \sum x^{2}-\left(\sum x\right)^{2}}{n(n-1)}
$$

When the statistical feature extraction is completed, the values are taken and the feature selection method is carried out. The statistical feature aids as the input to the feature selection method. With the selected feature the further classification is carried out."

$$
\text { Standard Deviation }(\sigma)=\sqrt{\frac{n \sum x^{2}-\left(\sum x\right)^{2}}{n(n-1)}}
$$

\section{FEATURE SELECTION USING J48 ALGORITHM}

J48 decision tree algorithm is adapted from the $\mathrm{C} 4.5$ algorithm in WEKA [13]. "It consists of a number of branches, one root, a number of nodes, and a number of leaves. One branch is a chain of nodes from the root to a leaf, and each node involves one attribute. The occurrence of an attribute in a tree provides information about the importance of the associated attribute [14].

A decision tree is a tree based knowledge representation methodology used to represent classification rules. J48 decision tree algorithm is a widely used one to construct decision trees [15]. The procedure of forming the decision tree and exploiting the same for feature selection is characterized by the following:

1. The set of features available at hand forms the input to the algorithm; the output is the decision tree.

2. The decision tree has leaf nodes, which represent class labels, and other nodes associated with the classes being classified.

3. The branches of the tree represent each possible value of the feature node from which they originate.

4. The decision tree can be used to classify feature vectors by starting at the root of the tree and moving through it until a leaf node, which provides a classification of the instance, is identified.

5. At each decision node in the decision tree, one can select the most useful feature for classification using appropriate estimation criteria. The criterion used to identify the best feature invokes the concepts of entropy reduction and information gain.

Information gain measures how well a given attribute separates the training examples according to their target classification.

The measure is used to select the candidate features at each step while growing the tree [16]. Information gain is the expected reduction in entropy caused by portioning the samples according to this feature.

Information gain $(\mathrm{S}, \mathrm{A})$ of a feature $\mathrm{A}$ relative to a collection of examples $\mathrm{S}$, is defined as:

$$
\operatorname{Gain}(S, A)=\operatorname{Entropy}(S)-\sum_{v \in \operatorname{Value}(A)} \times \frac{\left|S_{v}\right|}{|S|} \operatorname{Entropy}\left(S_{v}\right)
$$

where Value (A) is the set of all possible values for attribute $\mathrm{A}$, and $\mathrm{Sv}$ is the subset of $\mathrm{S}$ for which feature $\mathrm{A}$ has value $v$. Note the first term in the equation for gain is just the entropy of the original collection $\mathrm{S}$ and the second term is the expected value of the entropy after $\mathrm{S}$ is partitioned using feature A.

The expected entropy described by the second term is simply the sum of the entropies of each subset Sv, weighted by the fraction of samples $|\mathrm{Sv}| /|\mathrm{S}|$ that belong to $\mathrm{Sv}$. Gain (S, A) is, therefore, the expected reduction in entropy caused by knowing the value of feature A. Entropy is a measure of homogeneity of the set of examples and it is given by 


$$
\operatorname{Entropy}(S)=\sum_{i-1}^{c}-P_{i} \log _{2} P_{i}
$$

where, $\mathrm{c}$ is the number of classes, $\mathrm{Pi}$ is the proportion of $\mathrm{S}$ belonging to class ' $\mathrm{i}$ '.

The J48 decision tree algorithm has been applied to the problem of feature selection. The input to the algorithm is the set of statistical features described above and output of the decision tree shown in Figure 5.

It is clearly shown that the top node is the best node for classification. The other features in the nodes of decision tree perform in descending order of significance.

It is to be mentioned here that only features that contribute to the classification appear in the decision tree and other features do not contribute much. The features which have the less discriminating capability can be consciously discarded by deciding on the threshold.

This concept is made use for selecting good features. The algorithm identifies the good features for the purpose of classification of the given training data set, and thus reduces the domain knowledge required to select good features for pattern classification problem [17].

Referring from Figure 5, one can identify the most dominating features to represent the blade conditions are the sum, range, standard deviation, and kurtosis.

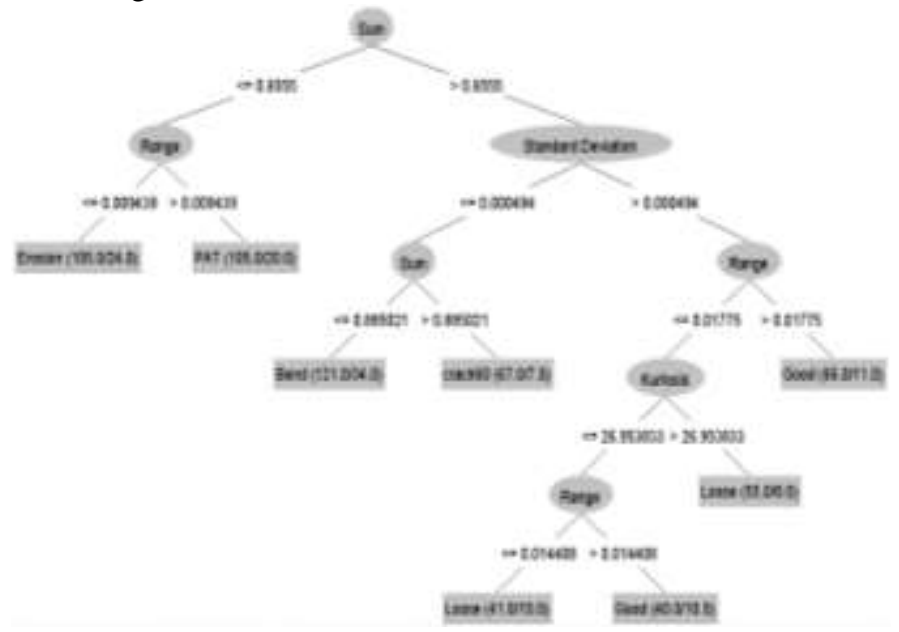

Figure 5: J48 Tree Classification for Feature Selection

\section{FEATURE CLASSIFICATION - ROTATION FOREST ALGORITHM}

Rotation Forest [18] is a new proposed strategy for building classifier ensembles utilizing individually prepared decision trees. "It was observed to be more exact than bagging, AdaBoost and random forest. Rotation forest lures upon the random forest views. The base classifiers are similar to individually made decision trees, yet in rotation forest every tree is trained on the full data set in a rotated feature space.

As the tree learning algorithm formulates the classification areas utilizing hyper planes parallel to the feature axes, a slight pivot of the axes might lead to an altered tree. The impact of turning the axes is that classification areas of high accuracy can be developed with less trees than in bagging and AdaBoost.

Random forest and bagging offers remarkable outcomes with very bulky ensembles having a huge number of estimator results in the enhancement of the accuracy of these approaches [19].
On the contrary, rotation forest is intended to work with a lesser number of ensembles. The quantity of trees necessary in the forest is commonly indicated by the user. Algorithm for rotation forest is as follows

- $\quad$ Let $T$ be the quantity of trees necessary to be built.

- Start with iterating from one over $T$, that is, we build $T$ trees.

For individual tree $\mathrm{T}$, perform the following steps:

- Split the features in the training set into $K$ nonoverlapping subcategories of equal size.

- $\quad$ We have $K$ datasets, each with $K$ features. For each of the $K$ datasets, we continue to do the following:

- Bootstrap $75 \%$ of the data from each $\mathrm{K}$ dataset and practice the bootstrapped illustration for further steps.

○ Run a principal component analysis (PCA) on the ith subset in K. Retain all the principal components. For each feature $\mathrm{j}$ in the Kth subset, we have a principle component, a. Let's indicate it as aij, where it's the principal component for the $\mathrm{jth}$ feature in the ith subset.

- Store the principal components for the subset.

- Create a rotation matrix of size, $n \times n$, where $n$ is the total number of features. Assemble the principal component in the matrix such that the components match the position of the feature in the original training dataset.

- Project the training dataset on the rotation matrix using the matrix multiplication.

- Construct a decision tree with the projected dataset.

- $\quad$ Store the tree and rotation matrix.

PCA is an unsupervised technique [20]. In multivariate statistical problems, PCA is used to decrease the dimension of the data with nominal data loss or, in other words, keep determined variation in the information.

In PCA, we find the directions in the information with the most variation, that is, the eigenvectors resultant to the prevalent eigenvalues of the covariance matrix and project the data onto these directions.

With a dataset $\left(\begin{array}{lll}n & x & m\end{array}\right)$ with $n$ instances and $m$ dimensions, PCA projects it onto a smaller subspace ( $\mathrm{n} x \mathrm{~d}$ ), where $\mathrm{d} \ll \mathrm{m}$."

\section{RESULTS AND DISCUSSION}

The vibration signals were recorded for good condition blade and other fault conditions of wind turbine blade. "Totally 600 samples were collected; out of which 100 samples were from good condition blade.

For other faults like blade bend, erosion, blade crack, hub-blade loose connection, pitch angle twist, 100 samples from each condition were collected.

The statistical features were extracted as features and serves as input to the algorithm. The corresponding condition of the classified data will be the required output of the algorithm [21-24]. 
Table 2: Confusion Matrix of Rotation Forest Algorithm

\begin{tabular}{|l|l|l|l|l|l|l|}
\hline $\begin{array}{l}\text { Blade } \\
\text { condition } \\
\text { s }\end{array}$ & $\begin{array}{l}\text { Goo } \\
\mathrm{d}\end{array}$ & $\begin{array}{l}\text { Ben } \\
\mathrm{d}\end{array}$ & $\begin{array}{l}\text { Crac } \\
\mathrm{k}\end{array}$ & $\begin{array}{l}\text { Loos } \\
\mathrm{e}\end{array}$ & $\begin{array}{l}\text { Pitc } \\
\mathrm{h} \\
\text { twist }\end{array}$ & $\begin{array}{l}\text { Erosio } \\
\mathrm{n}\end{array}$ \\
\hline Good & 88 & 0 & 1 & 11 & 0 & 0 \\
\hline Bend & 0 & 93 & 3 & 0 & 0 & 4 \\
\hline Crack & 0 & 3 & 94 & 3 & 0 & 0 \\
\hline Loose & 13 & 0 & 4 & 83 & 0 & 0 \\
\hline $\begin{array}{l}\text { Pitch } \\
\text { twist }\end{array}$ & 0 & 0 & 0 & 0 & 97 & 3 \\
\hline Erosion & 0 & 6 & 0 & 0 & 5 & 89 \\
\hline
\end{tabular}

The faults are created and compared with the good signal to predict whether the blade is faulty or not and the classifier performance is verified by 10 -fold cross validation [25-27]. In confusion matrix (Table 2), the diagonal elements denote the properly classified instances and the others are misclassified once. The classification accuracy of rotation forest algorithm is found to be $90.67 \%$. Table 1 represents the confusion matrix of the rotation forest classifier. From this classifier, the kappa statistic was found root mean square error value is very less about 0.1611 ."

Table 3: Class Wise Accuracy of Rotation Forest Algorithm

\begin{tabular}{|l|l|l|l|l|l|l|}
\hline Class & $\begin{array}{l}\text { TP } \\
\text { Rat } \\
\mathrm{e}\end{array}$ & $\begin{array}{l}\text { FP } \\
\text { Rate }\end{array}$ & $\begin{array}{l}\text { Precisio } \\
\mathrm{n}\end{array}$ & $\begin{array}{l}\text { Recal } \\
1\end{array}$ & $\begin{array}{l}\text { F- } \\
\text { Measur } \\
\mathrm{e}\end{array}$ & $\begin{array}{l}\text { ROC } \\
\text { area }\end{array}$ \\
\hline Good & $\begin{array}{l}0.8 \\
8\end{array}$ & $\begin{array}{l}0.02 \\
6\end{array}$ & 0.871 & 0.88 & 0.876 & $\begin{array}{l}0.97 \\
9\end{array}$ \\
\hline Bend & $\begin{array}{l}0.9 \\
3\end{array}$ & $\begin{array}{l}0.01 \\
8\end{array}$ & 0.912 & 0.93 & 0.921 & $\begin{array}{l}0.99 \\
4\end{array}$ \\
\hline Crack & $\begin{array}{l}0.9 \\
4\end{array}$ & $\begin{array}{l}0.01 \\
6\end{array}$ & 0.922 & 0.94 & 0.931 & $\begin{array}{l}0.99 \\
5\end{array}$ \\
\hline Loose & $\begin{array}{l}0.8 \\
3\end{array}$ & $\begin{array}{l}0.02 \\
8\end{array}$ & 0.856 & 0.83 & 0.843 & $\begin{array}{l}0.97 \\
6\end{array}$ \\
\hline $\begin{array}{l}\text { Pitch } \\
\text { twist }\end{array}$ & $\begin{array}{l}0.9 \\
7\end{array}$ & 0.01 & 0.951 & 0.97 & 0.96 & $\begin{array}{l}0.99 \\
9\end{array}$ \\
\hline $\begin{array}{l}\text { Erosio } \\
\text { n }\end{array}$ & $\begin{array}{l}0.8 \\
9\end{array}$ & $\begin{array}{l}0.01 \\
4\end{array}$ & 0.927 & 0.89 & 0.908 & $\begin{array}{l}0.99 \\
5\end{array}$ \\
\hline
\end{tabular}

The detailed class wise accuracy of the rotation forest algorithm is shown in Table 3.TP is used to predict the ratio of positives which are correctly classified as faults. "FP is commonly described as a false alarm in which the result that shows a given fault condition has been achieved when it really has not been achieved [28-31]. The true positive (TP) rate should be close to 1 and the false positive (FP) rate should be close to 0 for a better classifier. One can observe from Table 3, the TP rate of most of the classes are close to 1 and FP rate was close to 0 . This reassures that the result presented by confusion matrix in Table 4 .

Precision, recall, and f-measure are correlated. Precision is the probability of retrieved instances that are relevant to the class. That is, it is the ratio of true positive (TP) to the retrieved instances $(\mathrm{TP}+\mathrm{FP})$. It is expressed as $\frac{T P}{T P+F P}$. Precision is also called as the positive predictive value and can be defined as a measure of exactness or quality. The recall is the information retrieval is the probability of the faults that are relevant to the classification that was successfully retrieved. That is, the ratio of true positive (TP) to the overall instances $(\mathrm{TP}+\mathrm{FN})$. False negative $(\mathrm{FN})$ is considered as type 2 error in which the instances indicate the to be 0.888 with the mean absolute error to be 0.0574 . The

misclassification however, it is actually correctly classified. It is expressed as $\frac{T P}{T P+F N}$. The recall is also called as the measure of completeness or quantity [32-37].

\section{CONCLUSION}

The wind turbine is very important in the production of wind energy in day to day life. This paper presents an algorithmic based clarification of vibration signals for the assessment of wind turbine blade conditions. "From the acquired vibration data, a model was developed using data modelling technique. Rotation forest algorithm was used to learn and classify the different conditions of the blade. The model is tested under 10 -fold cross validation and correctly classified instances was found to be $90.67 \%$. The error rate is relatively less and may be considered for the blade fault diagnosis. Hence the rotation forest can be practically used for the condition monitoring of wind turbine blade to reduce the downtime and to provide more wind energy."

\section{REFERENCES}

1. Bellarmine GT, Urquhart J. Wind energy for the 1990s and beyond. Energy Conversion and Management. 1996 Dec 1; 37(12):1741-52.

2. Márquez FP, Tobias AM, Pérez JM, Papaelias M. Condition monitoring of wind turbines: Techniques and methods. Renewable Energy. 2012 Oct 1; 46:169-78.

3. Ciang CC, Lee JR, Bang HJ. Structural health monitoring for a wind turbine system: a review of damage detection methods. Measurement science and technology. 2008 Oct 13; 19(12):122001.

4. Kusiak A, Verma A. A data-driven approach for monitoring blade pitch faults in wind turbines. IEEE Transactions on Sustainable Energy. 2011 Jan; 2(1):8796.

5. Godwin JL, Matthews P. Classification and detection of wind turbine pitch faults through SCADA data analysis. IJPHM Special Issue on Wind Turbine PHM. 2013 Jul: 90.

6. Frost SA, Goebel K, Obrecht L. Integrating structural health management with contingency control for wind turbines. IJPHM Special Issue on Wind Turbine PHM (Color). 2013; 11.

7. Pratumnopharat P, Leung PS, Court RS. Wavelet transform-based stress-time history editing of horizontal axis wind turbine blades. Renewable energy. 2014 Mar $1 ; 63: 558-75$.

8. Herath MT, Lee AK, Prusty BG. Design of shapeadaptive wind turbine blades using Differential Stiffness Bend-Twist coupling. Ocean Engineering. 2015 Feb 1; 95:157-65.

9. Wang YZ, Li F, Zhang X, Zhang WM. Composite wind turbine blade aerodynamic and structural integrated design optimization based on RBF Meta-Model. In Materials Science Forum 2015 (Vol. 813, pp. 10-18). Trans Tech Publications.

10. Joshuva A, Sugumaran V. Fault diagnostic methods for wind turbine: A review. ARPN Journal of Engineering and Applied Sciences. 2016 Apr 11; 11(7):4654-4668.

11. Joshuva A, Sugumaran V. A data driven approach for condition monitoring of wind turbine blade using vibration signals through best-first tree algorithm and functional trees algorithm: A comparative study. ISA 
transactions. 2017 Mar 1; 67:160-172.

12. Joshuva A, Sugumaran V. Wind turbine blade fault diagnosis using vibration signals through decision tree algorithm. Indian Journal of Science and Technology. 2016 Dec 29; 9(48):1-7.

13. Joshuva A, Sugumaran V, Amarnath M. Selecting kernel function of Support Vector Machine for fault diagnosis of roller bearings using sound signals through histogram features. International Journal of Applied Engineering Research. 2015; 10(68):482-487.

14. Joshuva A, Sugumaran V. Wind Turbine Blade Fault Diagnosis Using Vibration Signals and Statistical Features through Nested Dichotomy Classifiers. International Journal of Pharmacy and Technology. 2017 Apr; 9(1): 29147-29164.

15. Joshuva A, Sugumaran V. Fault Diagnosis for Wind Turbine Blade through Vibration Signals Using Statistical Features and Random Forest Algorithm. International Journal of Pharmacy and Technology. 2017 Apr; 9(1): 28684-28696.

16. Joshuva, A., Sugumaran, V. Classification of Various Wind Turbine Blade Faults through Vibration Signals Using Hyperpipes and Voting Feature Intervals Algorithm. International Journal of Performability Engineering. 2017 May; 13: 247-258.

17. Joshuva A, Sugumaran V. Failure Analysis on Wind Blade Using Vibration Signals and Classifying the Failures Using Logit Boost Algorithm. International Journal of Control Theory and Applications. 2016; 9(52): 225-234.

18. Yadav AK, Chandel SS. Solar energy potential assessment of western Himalayan Indian state of Himachal Pradesh using J48 algorithm of WEKA in ANN based prediction model. Renewable Energy. 2015 Mar 1; 75:675-93.

19. Rodriguez JJ, Kuncheva LI, Alonso CJ. Rotation forest: A new classifier ensemble method. IEEE transactions on pattern analysis and machine intelligence. 2006 Oct; 28(10):1619-30.

20. Kuncheva LI, Rodríguez JJ. An experimental study on rotation forest ensembles. InInternational workshop on multiple classifier systems 2007 May 23 (pp. 459-468). Springer, Berlin, Heidelberg.

21. De Bock KW, Van den Poel D. An empirical evaluation of rotation-based ensemble classifiers for customer churn prediction. Expert Systems with Applications. 2011 Sep 15; 38(10):12293-301.

22. Joshuva A, Sugumaran V. Fault Diagnosis of Wind Turbine Blade Using Vibration Signals through J48 Decision Tree Algorithm and Random Tree Classifier. International Journal of Control Theory and Applications. 2016; 9(52):249-258.

23. Joshuva A, Sugumaran V. Multiclass Classifier Approach for Fault Diagnosis of Wind Turbine Blade Using Vibration Signals through Statistical Analysis. International Journal of Control Theory and Applications. 2016; 9(52): 235-247.

24. Joshuva A, Sugumaran V, Amarnath M, Lee SK. Remaining Life-Time Assessment of Gear Box Using Regression Model. Indian Journal of Science and Technology. 2016 Dec 28; 9(47):1-8.

25. Joshuva A, Sugumaran V. A Comparative Study for Condition Monitoring on Wind Turbine Blade using Vibration Signals through Statistical Features: A Lazy Learning Approach. International Journal of Engineering and Technology. 2018; 7(4.10):190-196.

26. Joshuva A, Sugumaran V. A Machine Learning Approach For Condition Monitoring Of Wind Turbine Blade Using Autoregressive Moving Average (ARMA) Features Through Vibration Signals: A Comparative
Study. Progress in Industrial Ecology-An International Journal. 2018; 12(1/2):14-34.

27. Joshuva A, Sugumaran V. A comparative study of Bayes classifiers for blade fault diagnosis in wind turbines through vibration signals. Struct Durab Health Monit (SDHM). 2017; 12(1):69-90.

28. More Vasudha, Panditrao Harshal, Joshuva A, Sugumaran V. Effect of Sampling Frequency and Sample Length on Fault Diagnosis of Wind Turbine Blade. Pakistan Journal of Biotechnology. 2018; 15(Special Issue ICRAME 17):14-17.

29. Joshuva A, Sugumaran V. A Study of Various Blade Fault Conditions on a Wind Turbine Using Vibration Signals through Histogram Features. Journal of Engineering Science and Technology. 2018 Jan; 13(1):102-121.

30. Manju BR, Joshuva A, Sugumaran V. A Data Mining Study for Condition Monitoring on Wind Turbine Blades Using Hoeffding Tree Algorithm Through Statistical and Histogram Features. International Journal of Mechanical Engineering and Technology. 2018; 9(1):1061-1079.

31. Deenadayalan, Sivakumar, S., Vishnuvardhan R., Sathish Kumar R. Fabrication and Characterisation of B-H-G Fiber with Teak Wood Particles Reinforced Hybrid Composite. International Journal of Engineering and Technology. 2018; 7(2.31): 208-211.

32. Sivakumar S, Dhanalakshmi V, Vishuvardhan R. Extraction of Subtractive Features of Prismatic Parts from STEP File for CAD/CAM Integration. Journal of Science and Technology, Malaysia. 2019 Jan; 27 (1): 343-356.

33. Vishnuvardhan R, Pooranam N, Sivakumar S, Vignesh T. Producing Electrical Energy from Light Intensity, Design Traking System by Dual Axis Method. International Journal of Mechanical and Production Engineering Research and Development (IJMPERD). 2019; Jan; 9: 503-509.

34. Joshuva A, Sugumaran V. Improvement in wind energy production through condition monitoring of wind turbine blades using vibration signatures and ARMA features: a data-driven approach. Progress in Industrial Ecology, an International Journal. 2019 Jun 21; 13(3):207-31.

35. Joshuva A, Sugumaran V. Selection of a meta classifierdata model for classifying wind turbine blade fault conditions using histogram features and vibration signals: a data-mining study. Progress in Industrial Ecology, an International Journal. 2019 Jun 21; 13(3):232-51

36. Joshuva A, Sugumaran V. Crack Detection and Localization on Wind Turbine Blade Using Machine Learning Algorithms: A Data Mining Approach. Struct Durab Health Monit (SDHM). 2019; 13(2):181-203.

37. Joshuva A, Sugumaran V. Fault Diagnosis and Localization of Wind Turbine Blade [dissertation], Vellore Institute of Technology, Chennai Campus. 2018. 